\title{
Pacific
}

Journal of

Mathematics

\section{THE INTRINSIC MOUNTAIN PASS}

MARTIN SCHECHTER

Volume $171 \quad$ No. 2

December 1995 


\title{
THE INTRINSIC MOUNTAIN PASS
}

\author{
MARTIN SCHECHTER
}

We show how the mountain pass and saddle point theorems can be formulated with out the use of "auxiliary" sets. Moreover, we show that results can still be obtained when some basic hypotheses of these theorems are not satisfied. We then apply our results to semilinear problems for partial differential equations.

\section{Introduction.}

In the mountain pass and saddle point theorems one is concerned with a $C^{1}$ functional $G$ on a Banach space $E$. One wishes to find a solution of $G^{\prime}(u)=0$ or at least a sequence $\left\{u_{k}\right\} \subset E$ such that

$$
G\left(u_{k}\right) \rightarrow c, G^{\prime}\left(u_{k}\right) \rightarrow 0
$$

for some $c \in R$. A general procedure was formulated in Brezis-Nirenberg $[\mathbf{B N}]$ as follows. One finds a compact metric space $K$ and selects a closed subset $K^{*}$ of $K$ such that $K^{*} \neq \phi, K^{*} \neq K$. One then picks a map $p^{*} \in$ $C\left(K^{*}, E\right)$ and defines

$$
\begin{gathered}
A=\left\{p \in C(K, E): \quad p=p^{*} \text { on } K^{*}\right\} \\
a=\inf _{p \in A} \max _{\xi \in K} G(p(\xi)) .
\end{gathered}
$$

Brezis-Nirenberg assume

(A) For each $p \in A, \max _{\xi \in K} G(p(\xi))$ is attained at a point in $K \backslash K^{*}$. They then prove that there is a sequence satisfying

$$
G\left(u_{k}\right) \rightarrow a, \quad G^{\prime}\left(u_{k}\right) \rightarrow 0 .
$$

In reference to the procedure one can ask three questions

1. Are the sets $K, K^{*}$ essential to the method, or can they be eliminated?

2. How can one verify $(A)$ ?

3. What can be said if $(A)$ fails to hold? 
The purpose of the present paper is to address these questions. Concerning the first, we show that indeed a quantity corresponding to (1.2) can be introduced which is "intrinsic" in nature and does not depend on the "auxiliary" sets $K, K^{*}$. For this purpose we use a definition given in [ST]. We define

$$
a=\inf _{\Gamma \in \Phi} \sup _{0 \leq s \leq 1, u \in A} G(\Gamma(s) u)
$$

where $A$ is a given subset of $E$ and $\Phi$ is a family of maps in $C(E \times[0,1], E)$ with certain properties (for a precise definition cf. Section 2 ). The quantity (1.4) depends only on $G$ and the set $A$. It is not required that $A=p^{*}\left(K^{*}\right)$ for some $K^{*}$ and $p^{*} \in C(K, E)$. In dealing with (1.4) we replace hypothesis (A) with

(B) For each $\Gamma \in \Phi$,

$$
\max _{0 \leq s \leq 1, u \in A} G(\Gamma(s) u)
$$

is attained at a point not in $A$.

We then show that hypothesis $(B)$ implies the existence of a sequence satisfying (1.3) (actually, we use a hypothesis weaker than $(B)$ ). From this it follows that

$$
a_{0}:=\sup _{A} G<a<\infty
$$

implies the existence of a sequence satisfying (1.3).

Concerning the second question, we show that hypothesis $(B)$ holds if and only if there is a subset $B$ of $E$ such that $A$ links $B$ in the sense of [ST] and

$$
a_{0}:=\sup _{A} G \leq b_{0}:=\inf _{B} G \text {. }
$$

The definition of linking given in [ST] differs from that usually found. Essentially, it says that $A$ and $B$ link if they cannot be "slipped" apart without intersection. In our opinion, this definition is more in keeping with the concept of linking (for a formal definition cf. Section 2). The results of the present paper show that the only practical way of applying hypothesis $(B)$ is to find a subset $B$ of $E$ such that $A$ links $B$ and (1.6) holds (the same is true for hypothesis $(A)$ ).

Concerning the third question, we note that the only case not covered is when there is a set $B$ such that $A$ links $B$ and

$$
-\infty<b_{0}<a_{0}=a<\infty .
$$

Surprisingly, something can be accomplished in this case as well. We have 
Theorem 1.1. Assume (1.7) and let

$$
B^{\prime}=\left\{v \in B: G(v)<a_{0}\right\} .
$$

Let $\alpha, T$ be positive numbers such that

$$
a_{0}-b_{0}<\alpha T, \quad T<d^{\prime}=d\left(A, B^{\prime}\right) .
$$

Then for each $\delta>0$ sufficiently small there is a $u \in E$ satisfying

$$
b_{0}-\delta \leq G(u) \leq a_{0}+\delta, \quad\left\|G^{\prime}(u)\right\| \leq \alpha
$$

and either

$$
d\left(u, B^{\prime}\right)<T \text { or } d\left(u, B \backslash B^{\prime}\right)<\delta / \alpha .
$$

Corollary 1.2. Let $\left\{B_{n}\right\}$ be a sequence of subsets of $E$ such that $A$ links $B_{n}$ for each $n$ and $d\left(A, B_{n}^{\prime}\right) \rightarrow \infty$, where

$$
B_{n}^{\prime}=\left\{v \in B_{n}: \quad G(v)<a_{0}\right\} .
$$

Assume that $a<\infty$ and that

$$
\inf _{B_{n}} G \geq b_{0}>-\infty
$$

Then there is a sequence $\left\{u_{k}\right\}$ satisfying (1.1) with $b_{0} \leq c \leq a_{0}$.

In essence, Corollary 1.2 says that if $A$ links $B_{n}$ for each $n$ and the part $B_{n}^{\prime}$ of $B_{n}$ on which $G$ is $<a_{0}$ moves out to infinity and (1.11) holds, then the mountain pass methods still apply. Special cases of this theorem were given in $[\mathbf{S c 1 - 4}, \mathbf{S i}]$. We present an application in Section 4.

\section{A Generalized Mountain Pass.}

Before stating our main theorems we recall the definition of linking sets given in [ST]. Let $E$ be a Banach space and Let $\Phi$ be the set of all continuous maps $\Gamma(t)$ from $E \times[0,1]$ to $E$ such that
(a) $\Gamma(0)=I$
(b) there is an $x_{0} \in E$ such that $\Gamma(1) x=x_{0}$ for each $x \in E$
(c) $\Gamma(t) x \rightarrow x_{0}$ as $t \rightarrow 1$ uniformly on bounded subsets of $E$
(d) for each $t \in[0,1), \Gamma(t)$ is a homeomorphism of $E$ onto itself and $\Gamma^{-1}$ is continuous on $E \times[0,1)$.


Definition. A subset $A$ of $E$ links a subset $B$ of $E$ if $A \cap B=\phi$ and for each $\Gamma \in \Phi$ there is a $t \in(0,1]$ such that $\Gamma(t) A \cap B \neq \phi$.

Let $A, B$ be subsets of a Banach space $E$ such that $A$ links $B$, and let $G$ be a $C^{1}$ functional on $E$. Define

$$
a:=\inf _{\Gamma \in \Phi} \sup _{0 \leq s \leq 1, u \in A} G(\Gamma(s) u), b_{0}=\inf _{B} G .
$$

Since $A$ links $B$, we have

$$
b_{0} \leq a
$$

Assume that

$$
d(A, B)>0
$$

Let

$$
B^{\prime}:=\{v \in B: G(v)<a\}
$$

We note that

$$
B^{\prime}=\phi \text { iff } b_{0}=a
$$

(If $D$ is any set, we write $d(D, \phi)=\infty$. Thus $d^{\prime}=\infty$ when $B^{\prime}=\phi$.) Let $\alpha, T$ be any positive numbers satisfying

$$
a-b_{0}<\alpha T, T<d^{\prime} .
$$

We have

Theorem 2.1. Assume in addition that

$$
-\infty<b_{0}, a<\infty
$$

Then for every $\delta>0$ sufficiently small there is a $u \in E$ such that

$$
b_{0}-\delta \leq G(u) \leq a+\delta, \quad\left\|G^{\prime}(u)\right\| \leq \alpha
$$

and either

$$
d\left(u, B^{\prime}\right)<T
$$

or

$$
d\left(u, B \backslash B^{\prime}\right)<\delta / \alpha .
$$


Corollary 2.2. If $b_{0}=a$, then there is a sequence $\left\{u_{k}\right\} \subset E$ such that

$$
G\left(u_{k}\right) \rightarrow a, G^{\prime}\left(u_{k}\right) \rightarrow 0, d\left(u_{k}, B\right) \rightarrow 0 .
$$

Corollary 2.3. Let $\left\{A_{n}\right\},\left\{B_{n}\right\}$ be sequences of subsets of $E$, and define

$$
a_{n}=\inf _{\Gamma \in \Phi} \sup _{0 \leq s \leq 1, u \in A_{n}} G(\Gamma(s) u), b_{o n}=\inf _{B_{n}} G
$$

$$
\begin{gathered}
a=\liminf a_{n}, b_{0}=\lim \sup b_{o n} \\
B_{n}^{\prime}=\left\{v \in B_{n}: G(v)<a_{n}\right\} \\
d_{n}^{\prime}=d\left(A_{n}, B_{n}^{\prime}\right) .
\end{gathered}
$$

Assume that $A_{n}$ links $B_{n}, d\left(A_{n}, B_{n}\right)>0$ and that

$$
-\infty<b_{0} \leq a<\infty
$$

Then there is a sequence $\left\{u_{k}\right\} \subset E$ such that

$$
G\left(u_{k}\right) \rightarrow c, b_{0} \leq c \leq a, G^{\prime}\left(u_{k}\right) \rightarrow 0 .
$$

Similarly we have

Theorem 2.4. Assume that $A, B \subset E, B$ links $A$, and $G \in C^{1}(E, R)$. Define

$$
\begin{gathered}
a_{0}:=\sup _{A} G, b:=\sup _{\Gamma \in \Phi} \inf _{0 \leq s \leq 1, v \in B} G(\Gamma(s) v) \\
A^{\prime}=\{u \in A: G(u)>b\}, d^{\prime \prime}=d\left(A^{\prime}, B\right)
\end{gathered}
$$

and let $\alpha, T$ be any positive constants satisfying

$$
a_{0}-b<\alpha T, T<d^{\prime \prime} \text {. }
$$

Assume also that

$$
-\infty<b, a_{0}<\infty
$$


and that (2.3) holds. Then for every $\delta>0$ sufficiently small there is a $u \in E$ satisfying

$$
b-\delta \leq G(u) \leq a_{0}+\delta,\left\|G^{\prime}(u)\right\| \leq \alpha
$$

and either

$$
d\left(u, A^{\prime}\right)<T
$$

or

$$
d\left(u, A \backslash A^{\prime}\right)<\delta / \alpha
$$

Corollary 2.5. Let $\left\{A_{n}\right\},\left\{B_{n}\right\}$ be sequences of subsets of $E$, and define

$$
a_{o n}=\sup _{A_{n}} G, b_{n}=\sup _{\Gamma \in \Phi} \inf _{0 \leq s \leq 1, v \in B_{n}} G(\Gamma(s) v)
$$

$$
\begin{gathered}
a_{0}=\liminf a_{o n}, b=\limsup b_{n} . \\
A_{n}^{\prime}=\left\{u \in A_{n}: G(u)>b_{n}\right\}, d_{n}^{\prime \prime}=d\left(A_{n}^{\prime}, B_{n}\right) .
\end{gathered}
$$

Assume that $B_{n}$ links $A_{n}, d\left(A_{n}, B_{n}\right)>0$ and that

$$
-\infty<b \leq a_{0}<\infty, d_{n}^{\prime \prime} \rightarrow \infty \text {. }
$$

Then there is a sequence $\left\{u_{k}\right\} \subset E$ such that

$$
G\left(u_{k}\right) \rightarrow c, b \leq c \leq a_{0}, G^{\prime}\left(u_{k}\right) \rightarrow 0 .
$$

Theorem 2.6. If $a_{0} \leq b_{0}$ and $a<\infty$, then there is a sequence $\left\{u_{k}\right\} \subset E$ such that

$$
G\left(u_{k}\right) \rightarrow a, G^{\prime}\left(u_{k}\right) \rightarrow 0
$$

We now show that we can remove the reference to the set $B$. We have Theorem 2.7. Assume that $a<\infty$ and that for each $\Gamma \in \Phi$ the set

$$
g_{\Gamma}:=\left\{v=\Gamma(s) u: s \in(0,1], u \in A, v \notin A, G(v) \geq a_{0}\right\}
$$


is not empty. Then there is a sequence satisfying (2.32).

Corollary 2.8. If $a<\infty$ and $a_{0} \neq a$, then there is a sequence satisfying (2.32).

Corollary 2.9. If $a<\infty$ and for each $\Gamma \in \Phi$

$$
\max _{0 \leq s \leq 1, u \in A} G(\Gamma(s) u)
$$

is attained at a point not in $A$, then there is a sequence satisfying (2.32).

We can summarize by

Theorem 2.10. Assume that $A$ links $B$ and that they satisfy (2.8). Then one of the following holds

(i) $a_{0} \neq a$

(ii) $b_{0}=a_{0}=a$

(iii) $b_{0}<a_{0}=a$.

In case (i) there is a sequence satisfying (2.32). In case (ii) there is a sequence satisfying (2.12). In case (iii) the conclusions of Theorem (2.1) hold.

Another consequence of Theorem 2.1 is

Theorem 2.11. Let $M, N$ be complementary subspaces of a Banach space $E$ with one of them being finite dimensional. Let $G$ be a $C^{1}$ functional on $E$, and define

$$
m_{0}:=\sup _{v \in N} \inf _{w \in M} G(v+w), m_{1}:=\inf _{w \in M} \sup _{v \in N} G(v+w) .
$$

Assume that

$$
-\infty<m_{0} \text { and } m_{1}<\infty
$$

Then there is a sequence $\left\{u_{k}\right\} \subset E$ such that

$$
G\left(u_{k}\right) \rightarrow c, m_{0} \leq c \leq m_{1}, G^{\prime}\left(u_{k}\right) \rightarrow 0 .
$$

Corollary 2.2 was proved in [ST] as well as Theorem 2.6. Theorem 2.11 generalizes theorems of $[\mathbf{S c 1}, \mathbf{S i}]$. The results of this section will be proved in Section 3.

Finally, we note the following 
Theorem 2.12. There is a $B \subset E$ such that $A$ links $B$ and $a_{0} \leq b_{0}$ if and only if $g_{\Gamma}$ defined by (2.33) is not empty for each $\Gamma \in \Phi$.

Remarks. There are basic differences between the approach of $[\mathbf{B N}]$ and that of $[\mathbf{S T}]$ and the present paper. The sets $K, K^{*}$ must be compact; otherwise their arguments fail. In $[\mathbf{S T}]$ the set $A$ neeed not be compact (or even closed). Also, both $K$ and $K^{*}$ must be chosen initially. In [ST] only the set $A$ is chosen. The sets (2.33) vary with $\Gamma$ and are determined automatically. For our linking, the set $B$ is only required to intersect the set (2.33) for each $\Gamma$. It is not required to intersect $p(K)$ for each continuous map $p$ (a much larger set). There are no counterparts of Theorem 2.1, Corollary 2.3, Theorem 2.4, Corollary 2.5 in the $[\mathrm{BN}]$ theory.

Assumption (2.3) is not required in Theorems 2.6, 2.7 and Corollary 2.9.

\section{The Flow.}

In this section we give the proofs of the results of Section 2 .

Proof of Theorem 2.1. Assume that the theorem is false. Then there are $\alpha, T$ satisfying (2.7) and a $\delta>0$ such that

$$
\left\|G^{\prime}(u)\right\|>\alpha
$$

when

$$
\begin{aligned}
u \in Q:=\left\{u \in E: b_{0}-3 \delta \leq\right. & G(u) \leq a+3 \delta \text { and either } \\
& \left.d\left(u, B^{\prime}\right) \leq T+3 \delta \text { or } d\left(u, B \backslash B^{\prime}\right) \leq 5 \delta / \alpha\right\} .
\end{aligned}
$$

Let

$$
\begin{aligned}
Q_{0}= & \left\{u \in E: b_{0}-2 \delta \leq G(u) \leq a+2 \delta\right. \\
& \text { and either } \left.d\left(u, B^{\prime}\right) \leq T+2 \delta \text { or } d\left(u, B \backslash B^{\prime}\right) \leq 4 \delta / \alpha\right\} \\
Q_{1}= & \left\{u \in E: b_{0}-\delta \leq G(u) \leq a+\delta \text { and either } d\left(u, B^{\prime}\right)\right. \\
\leq & \left.T+\delta \text { or } d\left(u, B \backslash B^{\prime}\right) \leq 3 \delta / \alpha\right\} .
\end{aligned}
$$

Define

$$
Q_{2}=E \backslash Q_{0}, \eta(u)=d\left(u, Q_{2}\right) /\left[d\left(u, Q_{1}\right)+d\left(u, Q_{2}\right)\right] .
$$

Note that $\eta=1$ on $Q_{1}, \eta=0$ on $\bar{Q}_{2}$ and $0<\eta<1$ otherwise. Let $d_{1}=$ $d\left(B \backslash B^{\prime}, A\right)$ and reduce $\delta$, if necessary, so that

$$
2 \delta<\alpha d_{1}, \delta<\alpha T-\left(a_{0}-b\right) .
$$


Let $\theta$ satisfy

$$
\frac{2}{3}<\theta<1,2 \delta<\theta \alpha d_{1}, \delta<\theta \alpha T-\left(a_{0}-b\right) .
$$

One can show that there is a locally Lipshitz continuous map $Y(u): \hat{E} \rightarrow$ $\hat{E}=\left\{u \in E: G^{\prime}(u) \neq 0\right\}$ satisfying

$$
\|Y(u)\| \leq 1,\left(G^{\prime}(u), Y(u)\right) \geq \theta\left\|G^{\prime}(u)\right\|, u \in \hat{E}
$$

(cf, e.g., $[\mathbf{B N}]$ ). Let $\sigma(t)$ be the flow generated by the vector field $\eta(u) Y(u)$ which is $I$ for $t=0$. Then

$$
\begin{aligned}
\|\sigma(t) u-u\| & =\left\|\int_{0}^{t} \eta(\sigma(\tau) u) Y(\sigma(\tau) u) d \tau\right\| \\
& \leq \Psi(t, u):=\int_{0}^{t} \eta(\sigma(\tau) u) d \tau, u \in E
\end{aligned}
$$

$G(\sigma(t) u)-G(u)=\int_{0}^{t} \eta(\sigma(\tau) u)\left(G^{\prime}(\sigma(\tau) u), Y(\sigma(\tau) u)\right) d \tau \geq \theta \alpha \psi(t, u), u \in E$.

Thus

$$
\|u-v\| \leq\|u-\sigma(t) v\|+\Psi(t, v) .
$$

If $u \in A$ and $v \in B^{\prime}$, this implies

$$
\|u-\sigma(t) v\| \geq d^{\prime}-T, 0 \leq t \leq T, u \in A, v \in B^{\prime} .
$$

On the other hand, if $v \in B \backslash B^{\prime}$, then $G(v) \geq a$ and $G(\sigma(t) v) \leq \max [G(v), a+$ $2 \delta$ ] by the definition of $\eta$. Hence by (3.7)

$$
\theta \alpha \Phi(t, v) \leq 2 \delta, t \geq 0, v \in B \backslash B^{\prime} .
$$

Consequently, by (3.8) - (3.10) we have

(3.11) $\|u-\sigma(t) v\| \geq \min \left[d^{\prime}-T, d_{1}-(2 \delta / \theta \alpha)\right]>0,0 \leq t \leq T, u \in A, v \in B$.

Let $B_{1}=\sigma(T) B$. I claim that $A$ links $B_{1}$. By (3.11), $A \cap \sigma(t) B=\phi$ for $0 \leq t \leq T$. Let $\Gamma \in \Phi$, and put $\Gamma_{1}(s)=\sigma(2 s T)^{-1}$ for $0 \leq s \leq \frac{1}{2}, \Gamma_{1}(s)=$ $\sigma(T)^{-1} \Gamma(2 s-1)$ for $\frac{1}{2}<s \leq 1$. Then $\Gamma_{1} \in \Phi$. Since $A$ links $B$, there is an $s_{1} \in[0,1]$ such that $\Gamma_{1}\left(s_{1}\right) A \cap B \neq \phi$. But $\sigma(2 s T)^{-1} A \cap B=\phi$ for $0 \leq s \leq \frac{1}{2}$ by (3.11). Thus $\frac{1}{2}<s_{1} \leq 1$, and $\sigma(T)^{-1} \Gamma\left(2 s_{1}-1\right) A \cap B \neq \phi$, or equivalently, 
$\Gamma\left(2 s_{1}-1\right) A \cap B_{1} \neq \phi$. Thus $A$ links $B_{1}$. Suppose there is a $t_{1} \leq T$ such that $\sigma\left(t_{1}\right) v \notin Q_{1}$. Then either

$$
G\left(\sigma\left(t_{1}\right) v\right)>a+\delta
$$

or

$$
d\left(\sigma\left(t_{1}\right) v, B^{\prime}\right)>T+\delta \text { and } d\left(\sigma\left(t_{1}\right) v, B \backslash B^{\prime}\right)>3 \delta / \alpha .
$$

But if $v \in B^{\prime}$, then $d\left(\sigma\left(t_{1}\right) v, B^{\prime}\right) \leq t_{1} \leq T$ by (3.6), and if $v \in B \backslash B^{\prime}$, then $d\left(\sigma\left(t_{1}\right) v, B \backslash B^{\prime}\right) \leq \Phi\left(t_{1}, v\right) \leq 2 \delta / \theta \alpha<3 \delta / \alpha$ by (3.6) and (3.10). Thus (3.13) is false if $v \in B$. Hence (3.12) must hold. This implies

$$
G(\sigma(T) v)>a+\delta
$$

On the other hand, if $\sigma(t) v \in Q_{1}$ for $0 \leq t \leq T$, then (3.7) gives

$$
G(\sigma(T) v) \geq b_{0}+\theta \alpha T>a+\delta
$$

Hence (3.14) holds for all $v \in B$. Thus means that

$$
\inf _{B_{1}} G \geq a+\delta \text {. }
$$

But by the definition of $a$, there is a $\Gamma \in \Phi$ such that

$$
\sup _{0 \leq s \leq 1, u \in A} G(\Gamma(s) u)<a+(\delta / 2)
$$

and since $A$ links $B_{1}$, there is an $s \in[0,1]$ such that $\Gamma(s) A \cap B_{1} \neq \phi$, and consequently (3.16) contradicts (3.15). Thus (3.1) cannot hold for $u$ satisfying (3.2), and the theorem is proved.

Proof of Corollary 2.2. In this case $B^{\prime}=\phi$ and $d^{\prime}=\infty$. For each $n$ we take $T_{n}=1, \delta_{n}=1 / n^{2}, \alpha_{n}=1 / n$ and apply Theorem 2.1 .

Proof of Corollary 2.3. For each $n$ we take $\delta_{n}=1 / n, T_{n}=d_{n}^{\prime} / 2, \alpha_{n}=$ $\left[\left(a_{n}-b_{o n}\right) / T_{n}\right]+\delta_{n}$. Then $\alpha_{n} \rightarrow 0$ and there is a $u_{n}$ such that

$$
b_{o n}-\delta_{n} \leq G\left(u_{n}\right) \leq a_{n}+\delta_{n},\left\|G^{\prime}\left(u_{n}\right)\right\| \leq \alpha_{n} .
$$

We take a renamed subsequence such that $a_{n} \rightarrow a, b_{o n} \rightarrow b_{0}$ and $G\left(u_{n}\right) \rightarrow c$.

Proof of Theorem 2.4. We interchange $A$ and $B$ and replace $G$ by $-G$ in Theorem 2.1. Then $b_{0}$ becomes $-a_{0}$ and $a$ becomes $-b$. $B^{\prime}$ becomes $A^{\prime}$. We then apply Theorem 2.1 . 
The proof of Corollary 2.5 in similar to that of Corollary 2.3.

Proof of Theorem 2.7. Let

$$
B=\bigcup_{\Gamma \in \Phi} g_{\Gamma}
$$

Then $A \cap B=\phi$ and for each $\Gamma \in \Phi$ there is a $v \in B$, an $s \in(0,1]$ and a $u \in A$ such that $\Gamma(s) u=v$. Hence

$$
\Gamma(s) A \cap B \neq \phi .
$$

Hence $A$ links $B$. Since $G(v) \geq a_{0}$ for each $v \in B$, we have $a_{0} \leq b_{0}$. We can now apply Theorem 2.6 to conclude that a sequence satisfying (2.32) exists.

Proof of Corollary 2.8. If $a_{0}<a$, then for each $\Gamma \in \Phi$ there is a $u \in A$ and an $s \in[0,1]$ such that $G(\Gamma(s) u)>a_{0}$. Clearly $v=\Gamma(s) u \notin A$. Thus $g_{\Gamma} \neq \phi$. We can now apply Theorem 2.7 .

Proof of Corollary 2.9. If the maximum (2.34) is attained at a point outside $A$, then this point is in $g_{\Gamma}$. Hence $g_{\Gamma}$ is not empty for all $\Gamma \in \Phi$. Apply Theorem 2.7.

Proof of Theorem 2.11. Assume, for definiteness, that $\operatorname{dim} N<\infty$. Let $\left\{\epsilon_{k}\right\}$ be a sequence tending to 0 . For each $k$ there are $v_{k} \in N, w_{k} \in M$ such that

$$
\inf _{M_{k}} G>m_{0}-\epsilon_{k}, \sup _{N_{k}} G<m_{1}+\epsilon_{k}
$$

where $M_{k}=v_{k} \oplus M$ and $N_{k}=w_{k} \oplus N$. Let

$$
A_{k}=\left\{v+w_{k}: v \in N,\left\|v-v_{k}\right\|=k\right\}, B_{k}=M_{k} .
$$

Note that $A_{k} \subset N_{k}$ and $A_{k} \cap B_{k}=\phi$. It is readily shown that $A_{k}$ links $B_{k}$ for each $k$ (cf., Propostion 1.2 of [ST]). By (3.18) we have

$$
b_{o k} \geq m_{0}-\epsilon_{k}, a_{o k} \leq m_{1}+\epsilon_{k} .
$$

Moreover, for each $k$,

$$
\Gamma_{k}(s) u=s\left(v_{k}+w_{k}\right)+(1-s) u
$$

is in $\Phi$. Consequently, definition (2.13) gives

$$
a_{k} \leq \sup _{N_{k}} G<m_{1}+\epsilon_{k} .
$$


Moreover, by (2.6)

$$
d_{k}^{\prime}=d\left(A_{k}, B_{k}^{\prime}\right) \geq d\left(A_{k}, B_{k}\right)=k \rightarrow \infty .
$$

If $a$ and $b_{0}$ are given by (2.14), we see that $m_{0} \leq b_{0}$ and $a \leq m_{1}$. We can now apply Corollary 2.3 to conclude that a sequence satisfying (2.37) exists. The proof for the case $\operatorname{dim} M<\infty$ is similar.

Proof of Theorem 2.12. The "if" part was already proved in the proof of Theorem 2.7. Conversely, suppose $A$ links $B$ and $a_{0} \leq b_{0}$. If $g_{\Gamma}=\phi$ for some $\Gamma \in \Phi$, then $\Gamma(s) A \cap B=\phi$ for each $s \in[0,1]$. This says that $A$ does not link $B$, a contradiction.

Remark. The proof of Theorem 2.6 was not given because it was proved in [ST]. However, it is a corollary of Theorem 2.1. To see this, note that if $a_{0} \leq b_{0}=a$, then $B^{\prime}=\phi, d^{\prime}=\infty$, and we can take $\alpha=\delta, T=1$. If $a_{0} \leq b_{0}<a$, let $B_{1}$ be given by (3.17). Then $A$ links $B_{1}$ and $a_{0} \leq b_{1} \equiv$ $\inf _{B_{1}} G=a$. We can now apply Theorem 2.1 again with $B$ replaced by $B_{1}$.

\section{An Application.}

In this section we show how the theorems of Section 2 can be applied. Let $\Omega$ be a smooth, bounded domain in $R^{n}$, and let $A$ be a selfadjoint operator on $L^{2}(\Omega)$ with discrete spectrum $0<\lambda_{0}<\lambda_{1}<\cdots<\lambda_{j}<\cdots$. We assume that $C_{0}^{\infty}(\Omega) \subset D:=D\left(A^{1 / 2}\right) \subset H^{m}(\Omega)$ for some $m>0(m$ need not be an integer). Let $q$ be a number satisfying

$$
2 \leq q<2 m /(n-2 m), 2 \leq q<\infty, 2 m<n ;
$$

and let $f(x, t)$ be a Caratheodory function on $\Omega \times R$. Assume

$$
|f(x, t)| \leq V_{0}(x)^{q}|t|^{q-1}+V_{0}(x) V_{1}(x)
$$

where $V_{0} \in L^{q}(\Omega), V_{1} \in L^{q^{\prime}}(\Omega)$ and multiplication by $V_{0}$ is a compact operator from $D$ to $L^{q}(\Omega)$.

(II) For some $\ell>0$ the function

$$
F(x, t):=\int_{0}^{t} f(x, s) d s
$$

satisfies

$$
\lambda_{\ell-1} t^{2}-W_{0}(x) \leq 2 F(x, t) \leq \nu t^{2}+V(x) p|t|^{p}+W_{1}(x)
$$

and

$$
\lambda_{\ell} t^{2}-W_{2}(x) \leq 2 F(x, t)
$$


where $\nu<\lambda_{\ell}, p>2$,

$$
B_{j}:=\int_{\Omega} W_{j}(x) d x<\infty, j=0,1,2
$$

and

$$
\|V u\|_{p}^{p} \leq C\left\|A^{1 / 2} u\right\|^{p}, u \in D .
$$

(III) For some $\mu>2$ the function

$$
H_{\mu}(x, t):=\mu F(x, t)-t f(x, t)
$$

satisfies

$$
H_{\mu}(x, t) \leq V_{3}(x)^{2} t^{2} \sigma(t)+W_{3}(x)
$$

where multiplication by $V_{3}$ is bounded from $D$ to $L^{2}(\Omega), W_{3} \in L^{1}(\Omega)$ and $\sigma(t)$ is a continuous function such that $\sigma(t) \rightarrow 0$ as $|t| \rightarrow \infty$.

(IV) $B_{0}+B_{1}<\left(1-\frac{2}{p}\right)\left(1-\frac{\nu}{\lambda_{\ell}}\right)^{p / p-2}\left(\frac{2}{p C}\right)^{2 / p-2}$.

We have

Theorem 4.1. Under hypotheses (I) - (IV) there is a solution of

$$
A u=f(x, u), \quad u \in D .
$$

Proof. Under hypothesis (I) the functional

$$
G(u)=a(u)-2 \int_{\Omega} F(x, u) d x
$$

has a continuous Frechet derivative on $D$ given by

$$
\left(G^{\prime}(u), v\right)=2 a(u, v)-2(f(\cdot, u), v)
$$

where

$$
a(u, v)=(A u, v), a(u)=a(u, u), u, v \in D .
$$

Let $N$ be the subspace spanned by the eigenfunctions corresponding to the eigenvalues $\lambda_{0}, \cdots, \lambda_{\ell-1}$, and let $M=N^{\perp} \cap D$, the orthogonal complement of $N$ on $D$. On $M$ we have

$$
G(w) \geq a(w)-\nu\|w\|^{2}-\|V w\|_{p}^{p}-B_{1} \geq\left(1-\frac{\nu}{\lambda_{\ell}}\right)\|w\|_{D}^{2}-C\|w\|_{D}^{p}-B_{1}
$$


by (4.2) and (4.5). If we take $\|w\|_{D}=\delta$ with $\delta^{p-2}=2\left(1-\frac{\nu}{\lambda_{\ell}}\right) / p$, we have

$$
G(w) \geq\left(1-\frac{2}{p}\right)\left(1-\frac{\nu}{\lambda_{\ell}}\right) \delta^{2}-B_{1} \geq \beta-B_{1},\|w\|_{D}=\delta
$$

where $\beta$ is the expression on the right hand side in hypothesis (IV). On the other hand, we have by (4.2)

$$
G(v) \leq a(v)-\lambda_{\ell-1}\|v\|^{2}+B_{0} \leq B_{0}, v \in N .
$$

Let $w_{0}$ be an eigenfunction corresponding to the eigenvalue $\lambda_{\ell}$ with unit norm, and let $N_{1}$ denote the subspace spanned by $N$ and $w_{0}$. By (4.3) we have

$$
G(v) \leq a(v)-\lambda_{\ell}\|v\|^{2}+B_{2} \leq B_{2}, v \in N_{1}
$$

Let

$$
\begin{aligned}
B & =\left\{w \in M:\|w\|_{D}=\delta\right\}, B_{n}=B \\
A_{1 n} & =\left\{v \in N:\|v\|_{D} \leq n\right\} \\
A_{2 n} & =\left\{u=v+s w_{0}: v \in N, s \geq 0,\|u\|_{D}=n\right\} .
\end{aligned}
$$

One can show that $A_{n}=A_{1 n} \cup A_{2 n}$ links $B_{n}=B$ for each $n$ (cf., [ST, Proposition 1.2]). By (4.11)

$$
b_{\text {on }}=b_{0} \geq \beta-B_{1}, b \geq-B_{1} .
$$

If $b_{0} \neq b$, then we can find a sequence in $D$ satisfying

$$
G\left(u_{k}\right) \rightarrow b, G^{\prime}\left(u_{k}\right) \rightarrow 0
$$

by Corollary 2.8. If $b_{0}=b$, then we have

$$
A_{n}^{\prime}=\left\{u \in A_{n}: G(u)>b\right\} \subset A_{2 n}
$$

since

$$
G(v) \leq B_{0}<\beta-B_{1}, v \in A_{1 n}
$$

by (4.12) and hypothesis (IV). Hence

$$
d_{n}^{\prime \prime}=d\left(A_{n}^{\prime}, B\right) \geq d\left(A_{2 n}, B\right) \geq n-\delta \rightarrow \infty .
$$

By (4.13), $a_{\text {on }} \leq B_{2}$ for each $n$. Hence we may apply Corollary 2.5 to conclude that there is a sequence in $D$ such that

$$
G\left(u_{k}\right) \rightarrow c, \beta-B_{1} \leq c \leq B_{2}, G^{\prime}\left(u_{k}\right) \rightarrow 0 .
$$


In either case we claim that the sequence is bounded in $D$. For suppose $\rho_{k}=\left\|u_{k}\right\|_{D} \rightarrow \infty$. Let $\tilde{u}_{k}=u_{k} / \rho_{k}$. Then by (4.15) or (4.16) we have

$$
\begin{aligned}
\left\|u_{k}\right\|_{D}^{2}-2 \int_{\Omega} F\left(x, u_{k}\right) d x & =0(1) \\
\left\|u_{k}\right\|_{D}^{2}-\int_{\Omega} f\left(x, u_{k}\right) u_{k} d x & =o\left(\rho_{k}\right) .
\end{aligned}
$$

Thus

$$
(\mu-2) \rho_{k}^{2}-2 \int_{\Omega} H\left(x, u_{k}\right) d x=o\left(\rho_{k}\right)
$$

Now

$$
\int \rho_{k}^{-2} H\left(x, u_{k}\right) d x \leq \int_{\Omega} V_{3}(x)^{2} \tilde{u}_{k}^{2} \sigma\left(\rho_{k} \tilde{u}_{k}\right) d x+\rho_{k}^{-2} \int_{\Omega} W_{3}(x) d x .
$$

There is a renamed subsequence for which $\tilde{u}_{k}(x)$ converges a.e. By hypothesis $V_{3} \tilde{u}_{k}$ is bounded in $L^{2}(\Omega)$ as well as $V_{3} \tilde{u}_{k} \sigma\left(u_{k}\right)$. Moreover, $V_{3} \tilde{u}_{k} \sigma\left(u_{k}\right) \rightarrow 0$ a.e. Hence the right hand side of (4.18) converges to 0. But this together with (4.17) implies that $\mu \leq 2$, contrary to assumption. Hence the sequence $\left\{u_{k}\right\}$ is bounded. It now follows by standard arguments that $\left\{u_{k}\right\}$ has a subsequence which converges in $D$ and that the limit satisfies $G^{\prime}(u)=0$ (cf., e.g., [Ra2]). It now follows form (4.9) that the limit is a solution of (4.7).

\section{References}

[BBF] P. Bartolo, V. Benci and D. Fortunato, Abstract critical point theorems and applications to some nonlinear problems with "strong" resonance at infinity, Nonlinear Anal. TMA, 7 (1983), 981-1012.

[BN] H. Brezis and L. Nirenberg, Remarks on finding critical points, Comm. Pure Appl. Math., 44 (1991), 939-964.

[E] I. Ekeland, On the variational principle, J. Math. Anal. Appl., 47 (1974), 324-353.

[Ra2] P.H. Rabinowitz, Minimax methods in critical point thoery with applications to differential equations, Cont. Board of Math. Sci. Reg. Cont. Ser. in Math., No. 65, Amer. Math. Soc., 1986.

[Sc1] M. Schechter, New saddle point theorems, Proceedings of an International Symposium on Generalized Functions and their Applications, Varanasli, India, December 23-26, 1991.

[Sc2] M. Schechter, A generalization of the saddle point method with applications, Ann. Polonici Math., 57 (1992), 269-281.

[Sc3] M. Schechter, Critıcal points over splitting subspaces, Nonlinearity, 6 (1993), 417427. 
[Sc4] M. Schechter, Splitting subspaces and critical points, Applicable Analysis, 49 (1993), 33-48.

[Si] E.A. de B.e. Silva, Linking theorems and applications to semilinear elliptic problems at resonance, Nonlinear Analysis TMA, 16 (1991), 455-477.

[ST] M. Schechter and K. Tintarev, Pairs of critical points produced by linking subsets with applications to semilinear elliptic problems, Bull. Soc. Math. Belg., 44 (1992), 249-261.

Received April 5, 1993 and revised January 19, 1994. Research supported in part by an NSF grant.

UNIVERSity OF CALIFORNIA

IRVINE, CA 92717

E-mail: mschecht@math.uci.edu 


\title{
PACIFIC JOURNAL OF MATHEMATICS
}

Founded by E. F. Beckenbach (1906-1982) and F. Wolf (1904-1989)

\section{EDITORS}

\author{
Sun-Yung Alice Chang (Managing Editor) \\ University of California \\ Los Angeles, CA 90095-1555 \\ pacific@math.ucla.edu
}

\section{F. Michael Christ}

University of California

Los Angeles, CA 90095-1555

christ@math.ucla.edu

Thomas Enright

University of California

San Diego, La Jolla, CA 92093

tenright@ucsd.edu

Nicholas Ercolani

University of Arizona

Tucson, AZ 85721

ercolani@math.arizona.edu
Robert Finn

Stanford University

Stanford, CA 94305

finn@gauss.stanford.edu

Vaughan F. R. Jones

University of California

Berkeley, CA 94720

vfr@math.berkeley.edu

Steven Kerckhoff

Stanford University

Stanford, CA 94305

spk@gauss.stanford.edu
Martin Scharlemann

University of California

Santa Barbara, CA 93106

mgscharl@math.ucsb.edu

Gang Tian

Courant Institute

New York University

New York, NY 10012-1100

tiang@taotao.cims.nyu.edu

V. S. Varadarajan

University of California

Los Angeles, CA 90095-1555

vsv@math.ucla.edu

\section{SUPPORTING INSTITUTIONS}

\section{CALIFORNIA INSTITUTE OF TECHNOLOGY \\ NEW MEXICO STATE UNIVERSITY \\ OREGON STATE UNIVERSITY \\ STANFORD UNIVERSITY \\ UNIVERSITY OF ARIZONA \\ UNIVERSITY OF BRITISH COLUMBIA \\ UNIVERSITY OF CALIFORNIA UNIVERSITY OF HAWAII}

\author{
UNIVERSITY OF MONTANA \\ UNIVERSITY OF NEVADA, RENO \\ UNIVERSITY OF OREGON \\ UNIVERSITY OF SOUTHERN CALIFORNIA \\ UNIVERSITY OF UTAH \\ UNIVERSITY OF WASHINGTON \\ WASHINGTON STATE UNIVERSITY
}

The supporting Institutions listed above contribute to the cost of publication of this Journal, but they are not owners or publishers and have no responsibility for its contents or policies.

Manuscripts must be prepared in accordance with the instructions provided on the inside back cover.

The Pacific Journal of Mathematics (ISSN 0030-8730) is published monthly except for July and August. Regular subscription rate: $\$ 215.00$ a year (10 issues). Special rate: $\$ 108.00$ a year to individual members of supporting institutions.

Subscriptions, orders for back issues published within the last three years, and changes of subscribers address should be sent to Pacific Journal of Mathematics, P.O. Box 4163, Berkeley, CA 94704-0163, U.S.A. Prior back issues are obtainable from Kraus Periodicals Co., Route 100, Millwood, NY 10546.

The Pacific Journal of Mathematics at the University of California, c/o Department of Mathematics, 981 Evans Hall, Berkeley, CA 94720 (ISSN 0030-8730) is published monthly except for July and August. Second-class postage paid at Berkeley, CA 94704, and additional mailing offices. POSTMASTER: send address changes to Pacific Journal of Mathematics, P.O. Box 6143, Berkeley, CA 94704-0163.

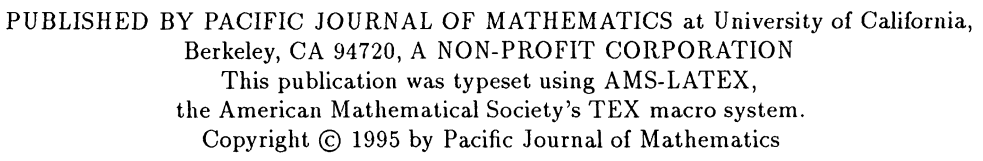




\section{PACIFIC JOURNAL OF MATHEMATICS}

\section{Volume $171 \quad$ No. $2 \quad$ December 1995}

On $H^{p}$-solutions of the Bezout equation

ERIC AMAR, JOAQUIM BRUNA FLORIS and ARTUR NiCOLAU

Amenable correspondences and approximation properties for von Neumann algebras

309

Claire ANANTHARAman-DElaroche

On moduli of instanton bundles on $\mathbb{P}^{2 n+1}$

VinCENZO ANCONA and Giorgio MaRia OtTAVIANI

Minimal surfaces with catenoid ends

JORGEN BERGLUND and WAYNE ROSSMAN

Permutation model for semi-circular systems and quantum random walks

PHILIPPE BIANE

The Neumann problem on Lipschitz domains in Hardy spaces of order less than one

RUSSELL M. BROWN

Matching theorems for twisted orbital integrals

409

REBECCA A. HERB

Uniform algebras generated by holomorphic and pluriharmonic functions on strictly

pseudoconvex domains

ALEXANDER IzZO

Quantum Weyl algebras and deformations of $U(g)$

NAIHUAN JING and JAMES ZHANG

Calcul du nombre de classes des corps de nombres

STÉPHANE LOUBOUTIN

On geometric properties of harmonic Lip ${ }_{1}$-capacity

PertTi MatTila and P. V. PARAmonov

Reproducing kernels and composition series for spaces of vector-valued holomorphic functions

Bent ØRsted and Genkai Zhang

Iterated loop modules and a filtration for vertex representation of toroidal Lie algebras

S. ESWARA RAO

The intrinsic mountain pass

MARTIN SCHECHTER

A Frobenius problem on the knot space

RON G. WANG

On complete metrics of nonnegative curvature on 2-plane bundles

DAVID YANG

Correction to: "Free Banach-Lie algebras, couniversal Banach-Lie groups, and more"

VLADIMIR G. PESTOV

Correction to: "Asymptotic radial symmetry for solutions of $\Delta u+e^{u}=0$ in a punctured disc"

KAI Seng (KAISing) CHOU (Tso) and Tom YAU-Heng WAN 